\title{
Three-Dimensional Optical Imaging Using Spectral Constraints
}

\author{
Subhadra Srinivasan, Hamid Dehghani, Brian W. Pogue \\ Thayer School of Engineering, Dartmouth College, Hanover NH 03755 \\ (Email: subha@dartmouth.edu, Tel:(603) 646-2119, Fax:(603) 646-3856) \\ Frederic Leblond and Xavier Intes \\ Advanced Research Techonologies (ART) Inc., 2300 Alfred Nobel Blvd, St. Laurent, QC-H4S2A4
}

\begin{abstract}
A novel scheme for implementing spectral constraints into a three-dimensional (3-D) image reconstruction model is presented. This approach, based on selecting measurements relating to spatial areas of maximum heterogeneity, makes spectral imaging computationally efficient and feasible in 3-D.

(C)2006 Optical Society of America

OCIS codes: (170.6960) Tomography
\end{abstract}

\subsection{Introduction}

Optical imaging has gained interest in the research and medical community due to its unique ability to obtain functional information from tissue in a non-invasive manner. Applied in the context of breast tissue, optical imaging in the near-infrared (NIR) region presents hemoglobin, oxygenation and density indices which enable separation of tissue types based on their metabolic status, as well as diagnosis of cancers[1, 2].

Conventionally, using measurements at the periphery of the breast, absorption and reduced scattering images were recovered using an appropriate model for light propagation[3]. The images of absorption and scatter were then spectrally de-convolved to yield images of total hemoglobin, oxygen saturation, water and scatter parameters. A recent significant advance in image reconstruction has permitted constraints using the known spectral shapes of chromophores and scatterers to be embedded into the image formation process[4, 5]. Using this approach, the functional images can be obtained directly, without recovery of optical coefficients. This approach, called the spectral method, has been shown to improve quantification, as well reduce cross-talk between different chromophores[4, 5], when applied in a two-dimensional (2-D) imaging setting.

A three-dimensional (3-D) model of tissue is known to provide a more accurate representation for light propagation in tissue. Absorption and scattering images have been recovered using a 3-D model in the past, albeit with under-estimated values[6]. Here, the spectral method has been extended to yield its advantages to 3-D. The spectral method, owing to its use of multi-wavelength measurements simultaneously, and recovery of five parameter images at once, presents certain computational challenges in inversion. Presented here is a novel scheme for applying the spectral method, where measurements are selected based on their effect on image reconstruction. Chosen based on a norm for the difference between model data and measured data, a reduced number of measurements (successfully capturing the most significant changes in the imaging domain) are used to recover functional images directly. This method presents an efficient solution to computational hassles relating to 3-D imaging while retaining the advantages of using spectral constraints in image reconstruction.

\subsection{Methods}

Based on the SoftScan breast optical imaging device developed by ART Advanced Research Technologies Inc. (ART) [7], a slab geometry has been used to generate measurements using the finite-element solver at Dartmouth. The SoftScan detection geometry consists in one source and five detectors with fixed relative positions (see Figure 1(a)). The source fiber is raster scanned through the entire imaging domain with a $3 \mathrm{~mm}$ resolution. A finite element model of the diffusion equation is used at Dartmouth, to model light propagation in tissue. Complete details of the implementation of this algorithm can be found elsewhere[3,6]. Briefly, the core of the reconstruction scheme is a Newton-Raphson minimization method for iteratively updating the chromophore concentrations and scattering. The method is based on minimization of the standard sum of squared differences between the measured and calculated optical radiance at specific detector locations. The spectral method yields the images of oxyhemoglobin $\left(\mathrm{HbO}_{2}\right)$, deoxyhemoglobin (Hb), water and scatter (scatter amplitude (a) and scatter power (b)). Details of its implementation in the 2-D case is in [5].

In order to efficiently process the data in 3-D, so that no memory hassles occur during the calculation of matrices for inversion, fewer lines of data points were used. A line (referred herein as a slice) of measurements consists in the data acquired from raster-scanning the source along $y$, for a fixed x. Figure 1(b) shows the difference between all measurement points and their division into slices. Typically 3 to 12 slices (out of 32) were selected with 


\section{MH8.pdf}

the criteria that they maximize the projection error: $\chi^{2}=\sum_{j=1}^{M}\left(\Phi_{\text {meas }}^{j}-\Phi_{\text {cal }}^{j}\right)^{2}$, where $\Phi_{\text {meas }}^{j}$ is the measured data and $\Phi_{\text {cal }}^{j}$ is the model calculated data for measurement point $j(=5 \times 32)$. In this paper we refer to this preferential use of selected measurements as the 'slice method'.

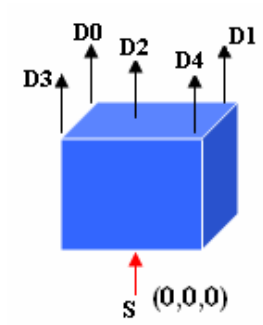

(a)
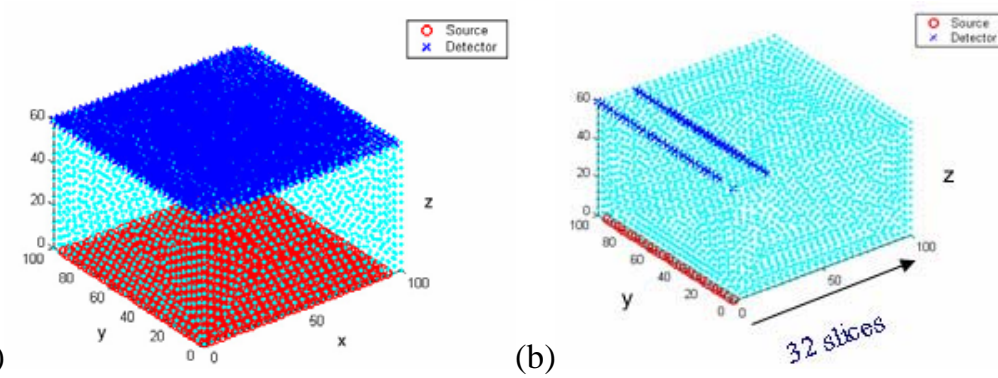

(c)

Figure 1: (a) source-detector configuration; for source $S$ at $(0.0 .0) \mathrm{mm}$, detectors are given as D0: $(-25,5,60), \mathrm{D} 1:(25,5,60), \mathrm{D} 2:(0,0,60)$, D3: $(-25,-15,60)$ and D4: $(25,-15,60) \mathrm{mm}$. (b) shows all source-detector positions within a domain mesh, when configuration in (a) is raster-scanned across a domain of $96 \times 96 \times 60 \mathrm{~mm}$ and (c) shows the division of the scan points into slices of data.

\subsection{Results:}

3.1 Validation of Slice Method: In order to compare the results of the slice method with the procedure using all available measurements, forward data was generated on a domain of size $60 \times 60 \times 60 \mathrm{~mm}$ with a single inclusion of diameter $15 \mathrm{~mm}$ centered at $(20,20,20) \mathrm{mm}$, on a finite element mesh containing 14,000 tetrahedral nodes. The inclusion had a contrast of 3:1 in $\mathrm{HbO}_{2}, 2: 1$ in $\mathrm{Hb}$, water and a and 20\% in b. The background concentrations were set to $\mathrm{HbO}_{2}: 14 \mu \mathrm{M}, \mathrm{Hb}: 8 \mu \mathrm{M}$, Water: $30 \%$, scatter amplitude, $a$ : 1.0 (units of $10^{-3 b}(\mathrm{~mm})^{b-1}$ ), scatter power, $b: 1.0$. Image reconstruction was carried out using the spectral method for the two cases, on a different mesh (9,000 nodes), and a cross-section of the recovered images for $\mathrm{HbO}_{2}$ and $\mathrm{Hb}$ are shown in figure 3. Both methods used the same regularization method and stopping criterion and the slice method used 6 slices out of total of 21 slices of data.

The inclusion is clearly visible in both cases and comparable in quantification. In Hb, a pseudo artifact is visible at the bottom of the slab, in the case using all measurements available. Preferential selection of data corresponding to the position of the main inclusion reduces this artifact. The images for water, scatter amplitude and scatter power (not shown here) were comparable for both methods. In order to compare the quantitative values, the maximum in the region of the inclusion is plotted for both methods in figure 3(f), which shows similar results for the two approaches. The same trend was found for average values in the background.

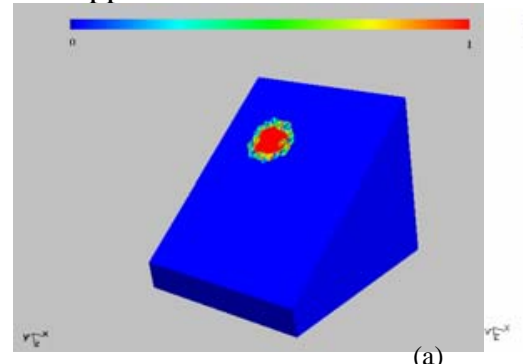

(a)

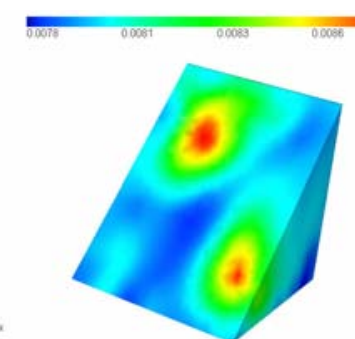

(d)

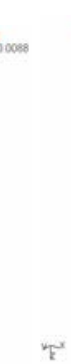

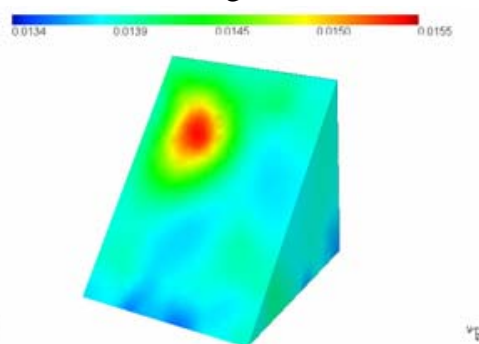

(b)

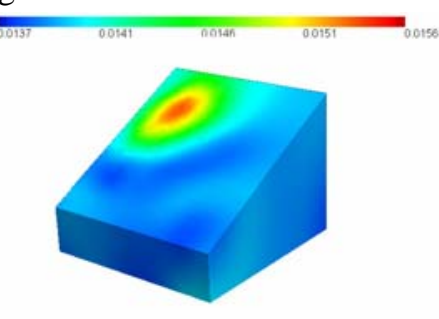

(c)

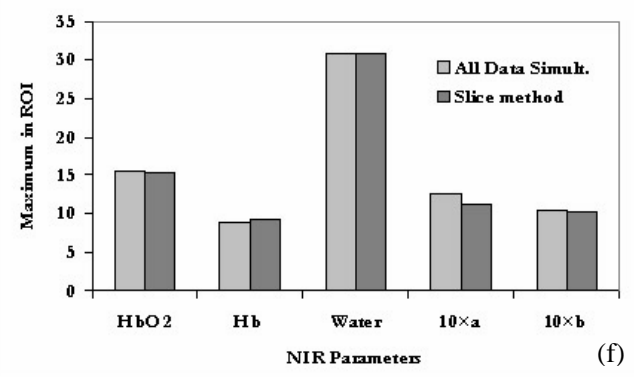

Figure 2: (a) cross-section of plane containing inclusion, scale shows region labels with 0 for background. (b) cross-section of $\mathrm{HbO} 2$ image using all data available (c) cross-section of $\mathrm{HbO}_{2}$ image using slice method, choosing slices of data corresponding to maximum projection error. (d) same as (b) for Hb. (e) same as (c) for Hb. All images displayed using Netgen. (f): maximum in inclusion for all parameters using both methods. 


\section{MH8.pdf}

3.2 Projection Error Criterion: With a homogeneous starting estimate for the five NIR parameters, calculating the difference between the corresponding forward data and the measured (or simulated on a different mesh) data yields the measurements probing spatial areas with maximum heterogeneity. If a tumor is present with a dominant contrast over the background, the projection error will have a maximum along the line of data corresponding to the plane of the tumor. In order to illustrate this, forward data from a different model based on the Rytov approximation implemented at ART[7] was used for image reconstruction. The data was generated on a test slab of size $96 \times 96 \times 60 \mathrm{~mm}$ with a single inclusion of size $15 \times 15 \times 20 \mathrm{~mm}$ centered at $(30,30,30) \mathrm{mm}$, having a contrast of 2:1 in $\mathrm{HbO} 2$ (all other images kept homogeneous). The background concentrations were given as $\mathrm{HbO}_{2}: 14 \mu \mathrm{M}, \mathrm{Hb}: 6$ $\mu \mathrm{M}$, Water: $30 \%$, Scatter amplitude: 0.8638 , Scatter power: 0.6 , based on typical breast properties. The source was raster-scanned in 3mm resolution along $\mathrm{x}$ and $\mathrm{y}$ axis giving 32 slices, each with 32 scan points.

After calibration to account for differences in the two models, the projection error was calculated using forward data on a homogeneous model with identical background properties with the finite element solver, and the data from ART. Figure 3(a) shows the projection error with slices. A maximum is expected at slice 10, corresponding to the position of the inclusion at $30 \mathrm{~mm}$ along $\mathrm{x}$-direction. A gaussian-shaped distribution of projection error with a maximum at slice 10 is observable. Using 3 slices of data based on the projection error criterion, the 3-dimensional images for $\mathrm{HbO}_{2}, \mathrm{Hb}$, water, scatter amplitude and scatter power were recovered. Shown in figure 3(b) is a cross-section of the images in the plane of inclusion. The inclusion is clearly visible in the expected position in $\mathrm{HbO}_{2}$ with other images staying almost homogeneous as expected.
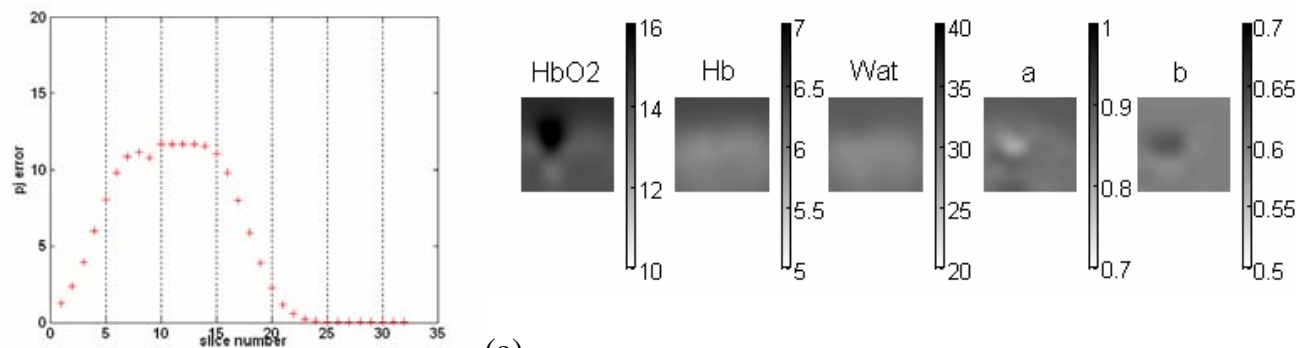

(a)

Figure 3: (a) projection error for different slices: slice 10 has maximum, corresponding to the location of the center of the inclusion. (b) crosssection of recovered $\mathrm{HbO}_{2}$ image using 3 lines of data, in plane of inclusion, shows increase at expected position.

\subsection{Discussion}

The spectral method has been found to provide accurate quantification in the breast with reduced cross-talk between chromophores[4,5]. This, coupled with a 3-D model for light propagation, provides one of the most accurate representations for breast imaging. The novel scheme of selecting the lines of data which have maximum effect on the imaging domain provides an efficient solution to the computational hassles associated with the method in 3-D. The results show that the use of this preferential selection of data provides similar quantification as using all available data. The method has been tested using measurements generated from the same model (finite element solver) as well as a different model (linear model at ART), and shows robust behavior for both. Preliminary studies on more complicated phantoms also indicate the same trend. In conclusion, the slice method is a powerful technique for implementing the spectral method in 3-D.

\subsection{Acknowledgements}

This work is funded by ART Inc.

\subsection{References}

1. Pogue, B.W., et al., Quantitative Hemoglobin Tomography with Diffuse Near-Infrared Spectroscopy: Pilot Results in the Breast. Radiology, 2001. 218(1): p. 261-6.

2. Tromberg, B.J., et al., Non-invasive in vivo characterization of breast tumors using photon migration spectroscopy. Neoplasia (New York), 2000. 2(1-2): p. 26-40.

3. Paulsen, K.D., and Jiang H., Spatially varying optical property reconstruction using a finite element diffusion equation approximation. Med. Phys., 1995. 22(6): p. 691-701.

4. $\quad$ Corlu, A., et al., Diffuse optical tomography with spectral constraints and wavelength optimization. Appl Opt, 2005. 44(11): p. 20822093.

5. Srinivasan, S., et al., Near-infrared characterization of breast tumors in-vivo using spectrally-constrained reconstruction. Technology in Cancer Research and Treatment, 2005. 4(5): p. 513-526.

6. $\quad$ Dehghani, H., et al., Three-dimensional optical-tomography: resolution in small-object imaging. Applied Optics, 2003. 42(16): p. 3117-3128.

7. Intes, X., et al., Time-Domain Optical Mammography SoftScan: Initial Results. Acad. Radiology, 2005. 12(8): p. 934-947. 